\title{
Constructing Tissuelike Complex Structures Using Cell-Laden DNA Hydrogel Bricks
}

\author{
Yijie Wang, ${ }^{\dagger}$ Yu Shao, ${ }^{\dagger}$ Xiaozhou Ma, ${ }^{\ddagger}$ Bini Zhou, ${ }^{\dagger}$ Alan Faulkner-Jones, ${ }^{\S}$ Wenmiao Shu, ${ }^{*}, \S$ \\ and Dongsheng Liu* ${ }^{*} \dagger$ (1)
}

${ }^{\dagger}$ Key Laboratory of Organic Optoelectronics and Molecular Engineering of the Ministry of Education, Department of Chemistry,
Tsinghua University, Beijing 100084, China
${ }^{\ddagger}$ School of Basic Medical Sciences, Lanzhou University, Lanzhou, Gansu 730000, China
${ }^{\S}$ Department of Biomedical Engineering, Faculty of Engineering, University of Strathclyde, Glasgow G4 0NW, United Kingdom

Supporting Information

ABSTRACT: Tissue engineering has long been a challenge because of the difficulty of addressing the requirements that such an engineered tissue must meet. In this paper, we developed a new "brick-to-wall" based on unique properties of DNA supramolecular hydrogels to fabricate threedimensional (3D) tissuelike structures: different cell types are encapsulated in DNA hydrogel bricks which are then combined to build 3D structures. Signal responsiveness of cells through the DNA gels was evaluated and it was discovered that the gel permits cell migration in 3D. The results demonstrated that this technology is convenient, effective and reliable for cell manipulation, and we believe that it will benefit artificial tissue fabrication and future large-scale production.

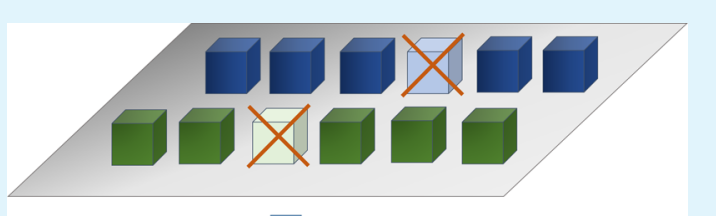

Bricks to Wall

KEYWORDS: DNA hydrogel, tissue engineering, cell arrangements, cell migration, self-healing, supramolecular

\section{INTRODUCTION}

Constructing artificial tissues or organs has long been a challenge in tissue engineering. ${ }^{1-3}$ To achieve this goal, many efforts have been made to arrange different types of living cells in a 3D manner. ${ }^{4-9}$ In 2012, Jiang and his co-workers developed a series of technologies to allocate different cells on PDMS film, then make a three-dimensional tubular structure by rolling the film up..$^{10,11}$ In 2015, Gatner et al. reported a method to attach different DNA sequences onto cells' surfaces through lipid insertion, followed by specific DNA recognition which leads to the fabrication of the multicellular organization of organoid-like tissues. ${ }^{12}$ Recently, 3D bioprinting technology has emerged as a promising, automated method to fabricate tissuelike structures. ${ }^{13-22}$ However, no single one of these strategies can meet the requirements of structural complexity, precision, cell viability and scalable fabrication alone. In this paper, we report a "brick-to-wall" technology to fabricate 3D tissue-like structures with multiple cell types at micrometer accuracy, which is based on the good biocompatibility and excellent self-healing property of the DNA supramolecular hydrogels. ${ }^{23-30}$ This stepwise building strategy can easily avoid damaging cells in the manufacturing process and is compatible with automatic manufacture processes. Cell behavior in the $3 \mathrm{D}$ hydrogel microenvironment, with surrounding signals and their cell-cell interactions are studied.

\section{RESULTS AND DISCUSSION}

As illustrated in Figure 1, the target cells were encapsulated within a pure DNA hydrogel and hydrogel/cell bricks were created either manually or by using 3D bioprinting. DNA hydrogels have been reported as possessing excellent biocompatibility, designable mechanical properties and comparable permeability to extracellular matrix (ECM), which can ensure the cell viability within a $3 \mathrm{D}$ environment. ${ }^{24,25}$ Also, the dynamic essence of DNA self-assembly through base-pairing confers the hydrogel's exceptional "self-healing" properties. ${ }^{26}$ By simply placing the cell containing hydrogel bricks together to form a structure, the adjacent bricks will start to fuse together within seconds and the boundary will disappear in minutes. Thus, 3D tissuelike structures could be easily achieved. It is generally acknowledged that all manufacturing processes will inevitably lead to errors and cell necrosis, and cause failure in next steps for tissue engineering. In our strategy, the cells' status is verified via microscopy before assembling the structure; thus, the bricks containing dead cells are excluded, which prevents defects in the final products.

As our previous work, ${ }^{24,30}$ two monomers, Y-shaped structures (Y) and linkers (L), which were consequences of

Received: February 2, 2017

Accepted: March 16, 2017

Published: March 16, 2017 


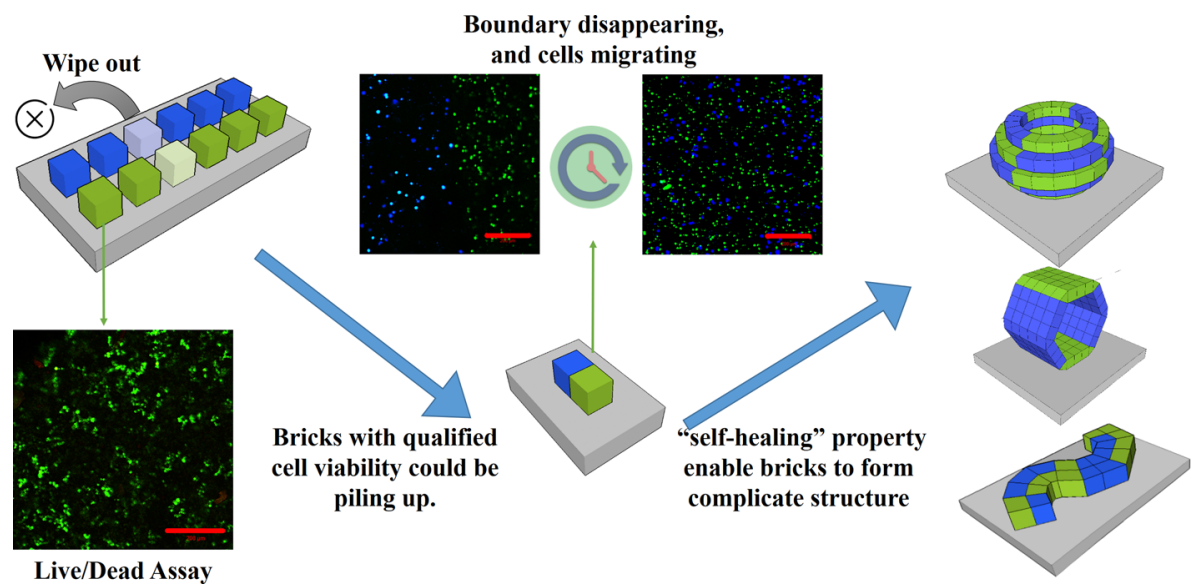

Figure 1. Scheme of brick-to-wall technology to fabricate 3D tissue-like structures. Different cell types are encapsulated in each DNA hydrogel brick and cultured for a period to eliminate damaged cells. The bricks are then combined to build the designed structures. Because the DNA hydrogel is capable of excellent self-healing, these cell-containing bricks could be combined and quickly merge into contiguous structures. Scale bars represent $200 \mu \mathrm{m}$.

complementation among D1, D2, D3 and D4, D5 single-strand DNAs (ssDNA) formed DNA hydrogels (Table S1). In a typical manufacturing procedure, the DNA hydrogels are prepared following previously reported protocol: scaffolds $\mathrm{Y}$ and $\mathrm{L}$ are preassembled in DMEM media and give a final concentration at $2.5 \mathrm{mM} \mathrm{Y}, 3.75 \mathrm{mM} \mathrm{L}$, respectively, and CellTrace-labeled HeLa cells are seeded in the Y-scaffold solution. ${ }^{25}$ These two solutions are mixed in equal volume to form the cell-containing hydrogel in a model to make a HeLa cell-containing, shaped hydrogel brick. Following the same procedure, other cells, such as THP-1 cells, can also be easily seeded in the hydrogel bricks. The cells are then cultured in the bricks for $48 \mathrm{~h}$, and their viabilities were examined by Live/ Dead assay. Results showed that the manufacturing process is very gentle to the cells and only small numbers of dead cells are found in all samples (Figure S1). The bricks containing insufficient viable cells could be easily abandoned at this stage. As the bricks are strong enough to be lifted by pipet or tweezers, two or more bricks could be easily attached together to make a designed structure and the adhesion process could be finished in seconds ${ }^{26}$ (Figure S2). These results proved that our strategy is feasible and reliable and can easily be conducted.

After piling the bricks together to make a 3D macro structure, cell migration is a key issue to build a structural organization. $^{31}$ With the cell-containing hydrogel bricks in hand, we used the Transwell migration protocol shown in Figure 2 to evaluate predictable cells behaviors triggered by signals in hydrogels. We employed hydrogels to replace Matrigel in simple Transwell cell behavior experiments, and used HeLa cells as a model object in the assay. The cells were cultured in $\mathrm{CO}_{2} /$ air $(5 \% / 95 \%)$ atmosphere at $37{ }^{\circ} \mathrm{C}$. The migration of the cells was monitored by fluorescence microscope. To verify that the hydrogel can permit transmigration of the cells, we filled the inset chamber with $100 \mu \mathrm{L}$ of DNA hydrogel formed with FBS-free DMEM medium; 100 $\mu \mathrm{L}$ of FBS-free DMEM HeLa suspensions $\left(0.5 \times 10^{6} / \mathrm{mL}\right)$ was then added. The lower compartment was filled with DMEM with $10 \%$ FBS to create the nutrient concentration gradient. As shown in Figure 2A, after $1 \mathrm{~h}$ of culture, there are no cells visible on the upper side of the membrane and very few cells are seen in the hydrogel (Figure S3). After $18 \mathrm{~h}$, a few cells could be seen on the upper side of the membrane. This
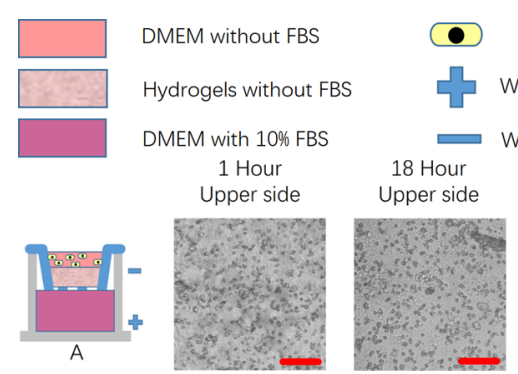

Hela cells

With $10 \%$ FBS
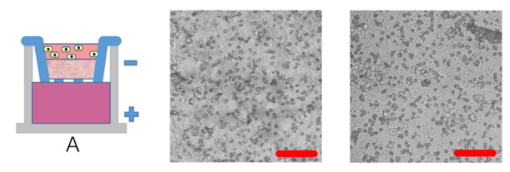
Lower side

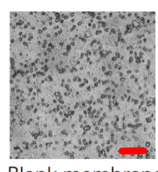

Blank membrane
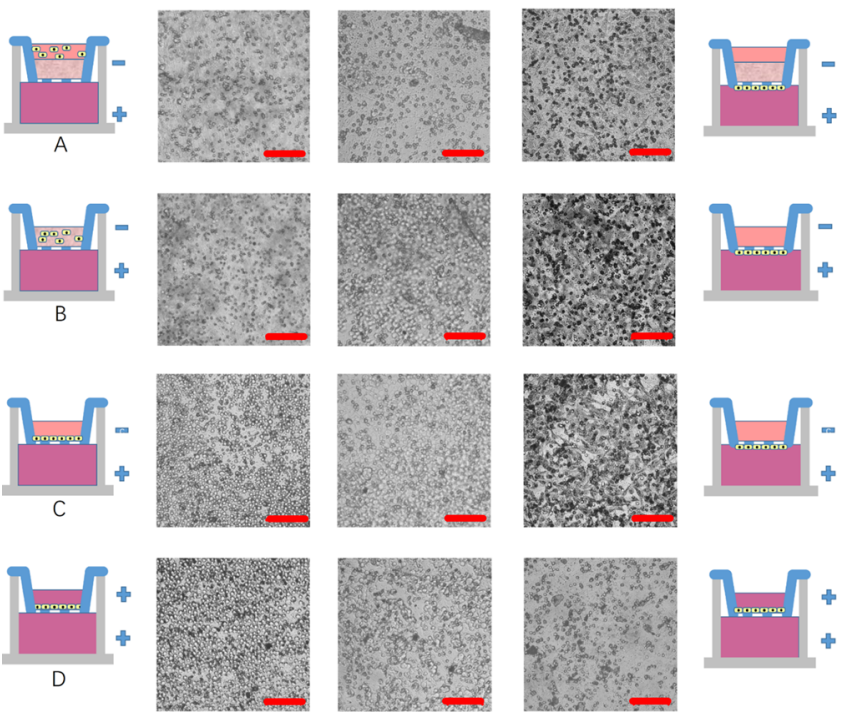

Figure 2. Illustration of the Transwell migration assay to evaluate signal-responsibility in DNA gels. The bottom of the inset is a microporous membrane, different culture systems can be set respectively in upper and lower compartments to facilitate the introduction of variables. (A) Cells on top of gels can migrate through the gel layer and membrane to the lower side. (B) Cells suspended within gels can migrate through the gel layer and membrane to the lower side. (C) Cells on top of the membrane without a gel layer can migrate through the membrane to lower side. $(\mathrm{A}-\mathrm{C})$ groups were set with nutritional gradient. (D) Group was set as control with no nutritional gradient, and the number of cells laid on the upper side of the membrane migrate to the lower side is low. Scale bars represent $100 \mu \mathrm{m}$.

suggests that the DNA hydrogel can permit cell migration at a reasonable speed (Figure S4). By embedding the cells in the hydrogel (Group B), we can see that the number of cells which reached the upper side of the membrane was significantly increased compared to group A after $18 \mathrm{~h}$. We proposed that 


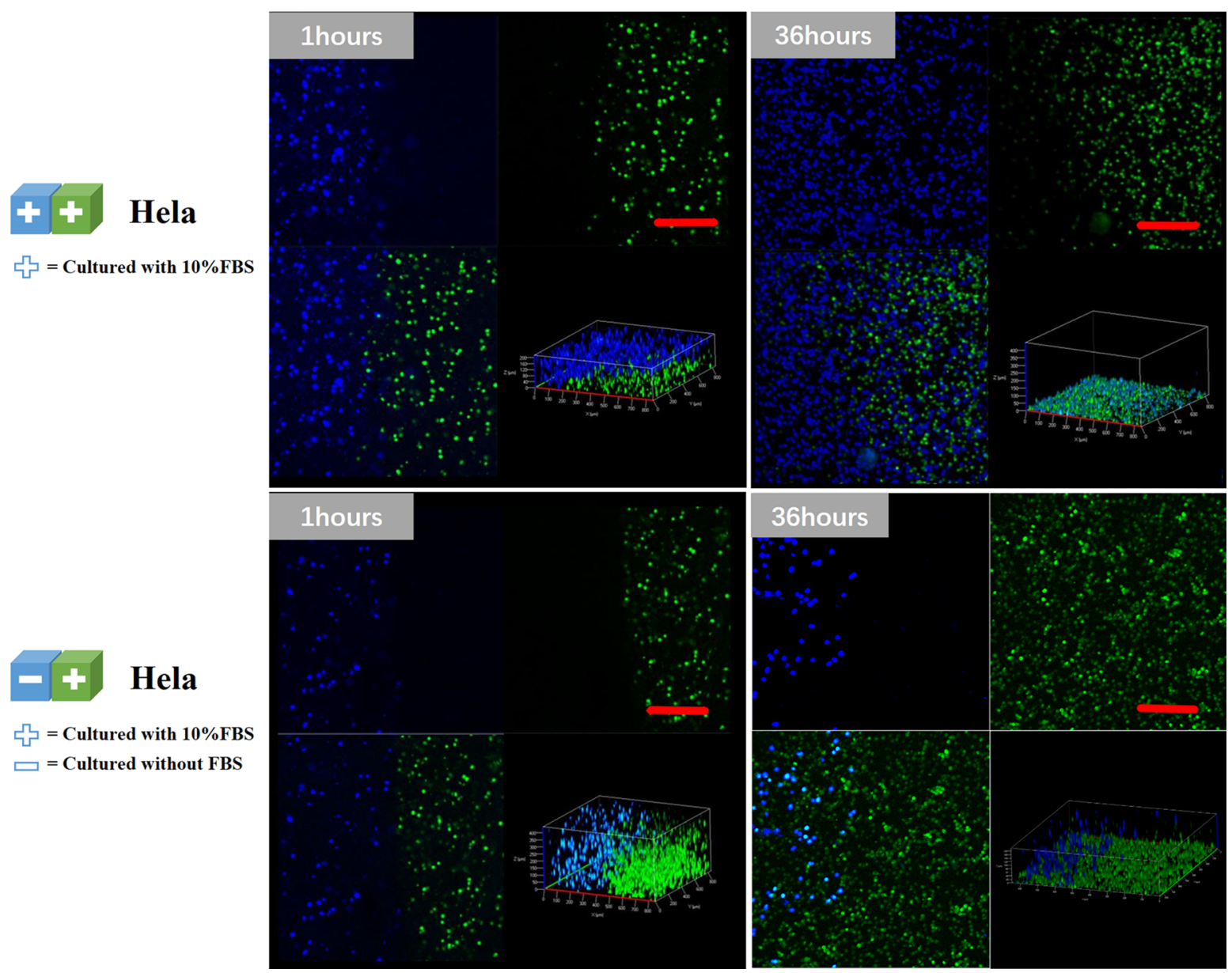

Figure 3. HeLa cells migration tracing in DNA gels. HeLa cells were labeled by blue and green living cell dye, CellTrace, and tracked by confocal microscopy. Gels with labeled cells were cultured and observed at 1 and $36 \mathrm{~h}$. The two bricks of gels were set with different nutrition conditions. Scale bars represent $100 \mu \mathrm{m}$.

this difference might be attributed to the longer transmigrating distance in Group A. If there is no hydrogel in the culture system, as shown in Figure $2 \mathrm{C}$ the upper surface of the membrane could be covered by cells in $1 \mathrm{~h}$ at a similar density to that shown at $18 \mathrm{~h}$ in group $\mathrm{B}$. This result proved that the hydrogel slows down the migration of the cells.

After $120 \mathrm{~h}$, the upper contents in the inset were removed; cells attached on the lower side of membrane are fixed with $2.5 \%$ glutaraldehyde, dehydrated in ethanol, and then observed by fluorescent microscope. In groups $\mathrm{A}, \mathrm{B}$, and $\mathrm{C}$, a large number of cells penetrated the membrane and were densely packed on the lower surface. To eliminate the possibility that gravity caused the penetration, we carried out a control experiment under the same condition as group $C$ but without the FBS gradient. As shown in Figure 2D, very few cells can be observed at the lower surface of the membrane. These results demonstrated that the DNA hydrogel is biocompatible and could provide an ECM-like environment for cell migration.

It is worth noting that this brick-to-wall strategy is suitable to build more complicated multicellular structures, and the DNA hydrogel provides sufficient cell mobility. We then used the technology to study the migration of cells between different hydrogel bricks. In the experiment, we employed live cell dyes, CellTrace, to label HeLa cells green $(495 / 519 \mathrm{~nm})$ and blue $(405 / 450 \mathrm{~nm})$ colors; after embedding the cells into two DNA hydrogel bricks and aligned the two bricks side-by-side, the two bricks fused immediately and then were cultured in atmosphere of $\mathrm{CO}_{2} /$ air $(5 \% / 95 \%)$ at $37{ }^{\circ} \mathrm{C}$. A confocal laser-scanning microscope was employed to study the cell distribution and migration at the interface. As shown in Figure 3, when two bricks both contain $10 \%$ FBS, a clear boundary could be seen within $1 \mathrm{~h}$. This result verified that the DNA hydrogel bricks can adhere effectively. In the following $36 \mathrm{~h}$ of culture, we saw that the cells noticeably migrated in two directions: downward, and laterally (perpendicular to the blocks' interface). These phenomena demonstrated that the interface might disappear and the self-healing between adjacent bricks is very efficient. The obvious downward migration of the cells could be attributed to the strong anchorage-dependence of HeLa cells. ${ }^{32}$

To find the possible driving force for the lateral movement, we removed the FBS from the bricks containing blue-labeled Hela cells. Following the same protocol, we can see that greenlabeled cells in the brick containing 10\% FBS exhibited stronger migration ability compared to the blue-labeled cells. After $36 \mathrm{~h}$, the green-labeled cells migrated into the space where the bluelabeled cells were initially seeded, but the blue-labeled cells mostly remained in their original position. This result illustrated that cell mobility can be adjusted by external signals in the DNA hydrogel system.

We also studied the behavior of THP-1 cells in a same manner. ${ }^{33}$ As a typical cell line with anchorage-independence, no obvious vertical migration was observed after $36 \mathrm{~h}$ (Figure 


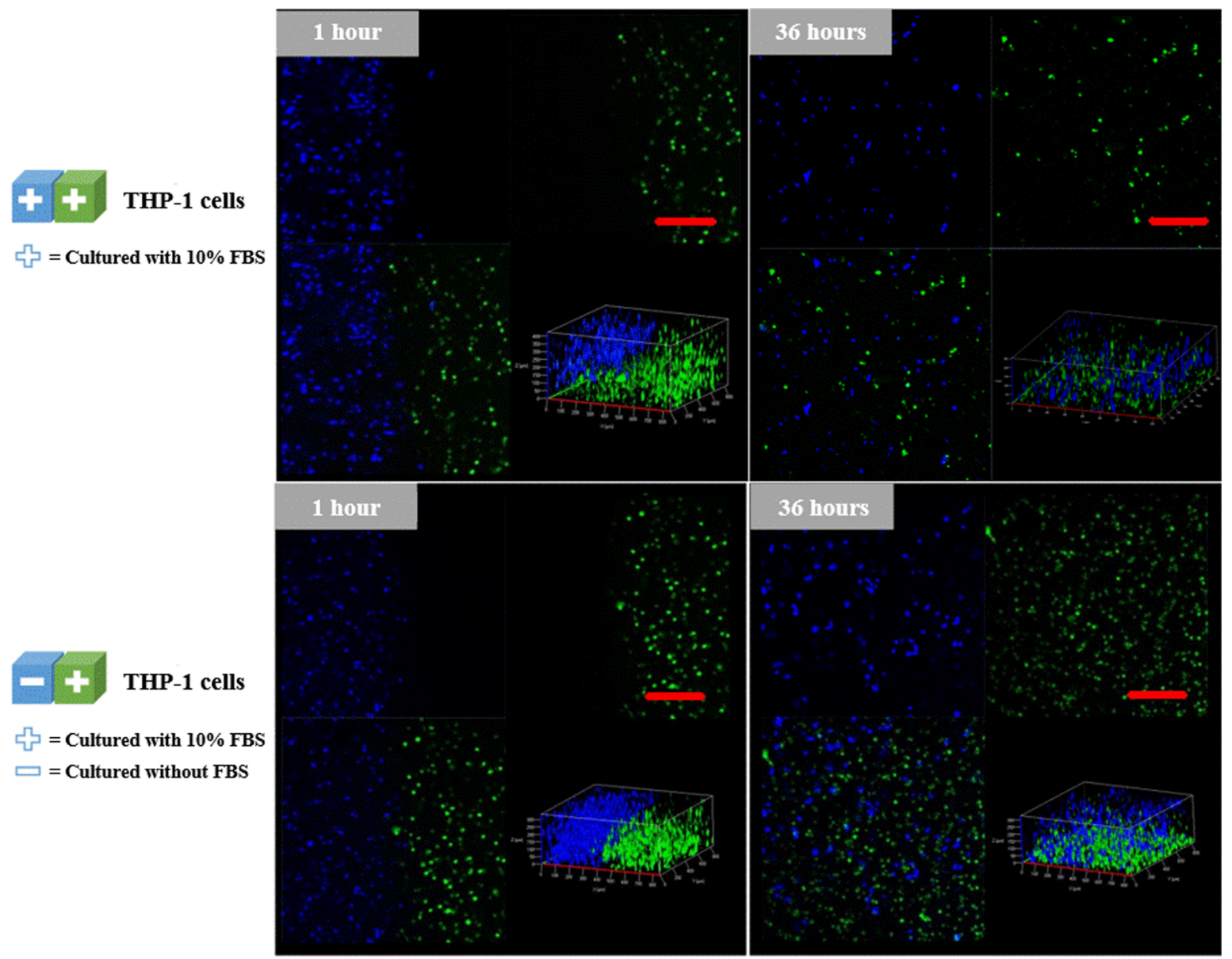

Figure 4. THP-1 cells migration tracing in gels. THP-1 cells were labeled by blue and green living cell dye, CellTrace, and tracked by confocal microscopy. Gels with labeled cells were cultured and observed in 1 and $36 \mathrm{~h}$. The two bricks of gels were set with different nutrition conditions. Scale bars represent $100 \mu \mathrm{m}$.

4). This result suggested that the vertical migration of HeLa cells might be caused by its anchorage-dependence. Also, the different FBS distribution showed no obvious influence on the cell migration, which is different with the behavior of HeLa cells but obvious lateral migrations were also been observed. These results further verified that the interfaces between DNA hydrogel bricks could be effectively eliminated by DNA chain exchange reactions, and the dynamic essence of DNA assemblies ensure the excellent cell permeability of the gels.

\section{CONCLUSION}

In summary, based on the outstanding self-healing property of DNA supramolecular hydrogels, we illustrated a brick-to-wall technology to fabricate $3 \mathrm{D}$ tissue-like structures. This strategy can be adapted to different cell types and can exclude damaged cells in the manufacturing process. Due to the mechanism, this technology can provide micrometer-resolution manufacturing and is compatible with automatic manufacture processes. Through the cell migration studies, we also demonstrated that the interface between hydrogel bricks could be eliminated by self-healing and does not influence the cell migration, which is crucial for the further development of artificial tissuelike structures. We believe this stepwise building strategy provides an innovative way to avoid cell damage during the manufacturing process and may enable the large-scale production of artificial tissue in the future.

\section{ASSOCIATED CONTENT}

\section{Supporting Information}

The Supporting Information is available free of charge on the ACS Publications website at DOI: 10.1021/acsami.7b01604.

Experimental details, confocal data, information on DNA sequences, and the Transwell experiment data (PDF)

\section{AUTHOR INFORMATION}

\section{Corresponding Authors}

*E-mail: will.shu@strath.ac.uk.

*E-mail: liudongsheng@tsinghua.edu.cn.

\section{ORCID}

Dongsheng Liu: 0000-0002-2583-818X

\section{Notes}

The authors declare no competing financial interest.

\section{ACKNOWLEDGMENTS}

We thank Associate Professor Mou's group in Lanzhou University for their assistant in cell culture and related experiments. We also thank National Basic Research Program of China (973 program, No. 2013CB932803), the National Natural Science Foundation of China (No. 21534007, 91427302, 21421064). AFJ and WS acknowledge the funding support from UK's EPSRC (EP/M506837/1, EP/P511420/1). 


\section{REFERENCES}

(1) Langer, R.; Vacanti, J. P. Tissue Engineering. Science 1993, 260, 920-926.

(2) Khademhosseini, A.; Vacanti, J. P.; Langer, R. Progress in Tissue Engineering. Sci. Am. 2009, 300, 64-71.

(3) Khademhosseini, A.; Langer, R. A Decade of Progress in Tissue Engineering. Nat. Protoc. 2016, 11, 1775-1781.

(4) Wang, Z.; Zhang, F.; Wang, Z.; Liu, Y.; Fu, X.; Albert, J.; Yung, B. C.; Chen, W.; Fan, J.; Yang, X.; Niu, G.; Chen, X. Hierarchical Assembly of Bioactive Amphiphilic Molecule Pairs into Supramolecular Nanofibril Self-Supportive Scaffolds for Stem Cell Differentiation. J. Am. Chem. Soc. 2016, 138, 15027-15034.

(5) Silva, L. P. P.; Pirraco, R. P. P.; Santos, T. C. C.; Novoa-Carballal, R.; Cerqueira, M. T.; Reis, R. L.; Correlo, V. M.; Marques, A. P. Neovascularization Induced by the Hyaluronic Acid-Based SpongyLike Hydrogels Degradation Products. ACS Appl. Mater. Interfaces 2016, 8, 33464-33474.

(6) Cohen, D. L.; Malone, E.; Lipson, H.; Bonassar, L. J. Direct Freeform Fabrication of Seeded Hydrogels in Arbitrary Geometries. Tissue Eng. 2006, 12, 1325-1335.

(7) Zheng, W.; Zhang, W.; Jiang, X. Precise Control of Cell Adhesion by Combination of Surface Chemistry and Soft Lithography. Adv. Healthcare Mater. 2013, 2, 95-108.

(8) Li, Y.; Yuan, B.; Ji, H.; Han, D.; Chen, S.; Tian, F.; Jiang, X. A Method for Patterning Multiple Types of Cells by Using Electrochemical Desorption of Self-Assembled Monolayers within Microfluidic Channels. Angew. Chem., Int. Ed. 2007, 46, 1094-1096.

(9) Li, P.; Yin, Z.; Dou, X-Q. Q.; Zhou, G.; Feng, C-L. L. Convenient Three-Dimensional Cell Culture in Supermolecular Hydrogels. ACS Appl. Mater. Interfaces 2014, 6, 7948-7952.

(10) Zheng, W.; Jiang, X. Precise Manipulation of Cell Behaviors on Surfaces for Construction of Tissue/organs. Colloids Surf., B 2014, 124, 97-110.

(11) Yuan, B.; Jin, Y.; Sun, Y.; Wang, D.; Sun, J.; Wang, Z.; Zhang, W.; Jiang, X. Strategy for Depositing Different Types of Cells in Three Dimensions to Mimic Tubular Structures in Tissues. Adv. Mater. 2012, 24, 890-896.

(12) Todhunter, M. E.; Jee, N. Y.; Hughes, A. J.; Coyle, M. C.; Cerchiari, A.; Farlow, J.; Garbe, J. C.; LaBarge, M. A.; Desai, T. A.; Gartner, Z. J. Programmed Synthesis of Three-Dimensional Tissues. Nat. Methods 2015, 12, 975-981.

(13) Groll, J.; Boland, T.; Blunk, T.; Burdick, J. A.; Cho, D.-W. W.; Dalton, P. D.; Derby, B.; Forgacs, G.; Li, Q.; Mironov, V. A.; Moroni, L.; Nakamura, M.; Shu, W.; Takeuchi, S.; Vozzi, G.; Woodfield, T. B.; Xu, T.; Yoo, J. J.; Malda, J. Biofabrication: Reappraising the Definition of an Evolving Field. Biofabrication 2016, 8, 013001.

(14) Murphy, S. V.; Atala, A. 3D Bioprinting of Tissues and Organs. Nat. Biotechnol. 2014, 32, 773-785.

(15) Faulkner-Jones, A.; Fyfe, C.; Cornelissen, D.-J. J.; Gardner, J.; King, J.; Courtney, A.; Shu, W. Bioprinting of Human Pluripotent Stem Cells and Their Directed Differentiation into Hepatocyte-like Cells for the Generation of Mini-Livers in 3D. Biofabrication 2015, 7, 044102.

(16) Li, C.; Faulkner-Jones, A.; Dun, A. R.; Jin, J.; Chen, P.; Xing, Y.; Yang, Z.; Li, Z.; Shu, W.; Liu, D.; Duncan, R. R. Rapid Formation of a Supramolecular Polypeptide-DNA Hydrogel for in Situ ThreeDimensional Multilayer Bioprinting. Angew. Chem., Int. Ed. 2015, 54, 3957-3961.

(17) Wang, M.; Vorwald, C.; Dreher, M.; Mott, E.; Cheng, M.; Cinar, A.; Mehdizadeh, H.; Somo, S.; Dean, D.; Brey, E.; Fisher, J. Evaluating 3D-Printed Biomaterials as Scaffolds for Vascularized Bone Tissue Engineering. Adv. Mater. 2015, 27, 138-144.

(18) Kolesky, D. B.; Truby, R. L.; Gladman, A. S.; Busbee, T. A.; Homan, K. A.; Lewis, J. A. 3D Bioprinting of Vascularized, Heterogeneous Cell-Laden Tissue Constructs. Adv. Mater. 2014, 26, 3124-3130.

(19) Hölzl, K.; Lin, S.; Tytgat, L.; Van Vlierberghe, S.; Gu, L.; Ovsianikov, A. Bioink Properties before, during and after 3D Bioprinting. Biofabrication 2016, 8, 032002.
(20) Tabriz, A. G.; Hermida, M. A.; Leslie, N. R.; Shu, W. Threedimensional Bioprinting of Complex Cell Laden Alginate Hydrogel Structures. Biofabrication 2015, 7, 045012.

(21) Ouyang, L.; Yao, R.; Chen, X.; Na, J.; Sun, W. 3D printing of HEK 293FT Cell-Laden Hydrogel into Macroporous Constructs with High Cell Viability and Normal Biological Functions. Biofabrication 2015, 7, 015010.

(22) Di Luca, A.; Longoni, A.; Criscenti, G.; Mota, C.; van Blitterswijk, C.; Moroni, L. Toward Mimicking the Bone Structure: Design of Novel Hierarchical Scaffolds with a Tailored Radial Porosity Gradient. Biofabrication 2016, 8, 045007.

(23) Cheng, E.; Xing, Y.; Chen, P.; Yang, Y.; Sun, Y.; Zhou, D.; Xu, L.; Fan, Q.; Liu, D. A pH-Triggered, Fast-Responding DNA Hydrogel. Angew. Chem., Int. Ed. 2009, 48, 7660-7663.

(24) Xing, Y.; Cheng, E.; Yang, Y.; Chen, P.; Zhang, T.; Sun, Y.; Yang, Z.; Liu, D. Self-Assembled DNA Hydrogels with Designable Thermal and Enzymatic Responsiveness. Adv. Mater. 2011, 23, 11171121.

(25) Jin, J.; Xing, Y.; Xi, Y.; Liu, X.; Zhou, T.; Ma, X.; Yang, Z.; Wang, S.; Liu, D. A Triggered DNA Hydrogel Cover to Envelop and Release Single Cells. Adv. Mater. 2013, 25, 4714-4717.

(26) Li, C.; Chen, P.; Shao, Y.; Zhou, X.; Wu, Y.; Yang, Z.; Li, Z.; Weil, T.; Liu, D. A Writable Polypeptide-DNA Hydrogel with Rationally Designed Multi-Modification Sites. Small 2015, 11, $1138-1143$.

(27) Li, C.; Zhou, X.; Shao, Y.; Chen, P.; Xing, Y.; Yang, Z.; Li, Z.; Liu, D. A Supramolecular Hydrogel with Identical Cross-Linking Point Density but Distinctive Rheological Properties. Mater. Chem. Front. 2017, DOI: $10.1039 / \mathrm{C} 6 \mathrm{QM} 00176 \mathrm{~A}$.

(28) Zhou, X.; Li, C.; Shao, Y.; Chen, C.; Yang, Z.; Liu, D. Reversibly Tuning the Mechanical Properties of a DNA Hydrogel by a DNA Nanomotor. Chem. Commun. 2016, 52, 10668-10671.

(29) Shi, J.; Jia, H.; Liu, D. pH-Responsive Supramolecular Hydrogel Based on One Short Strand DNA. Acta Polym. Sin. 2017, 1, 135-142.

(30) Shao, Y.; Jia, H.; Cao, T.; Liu, D. Supramolecular Hydrogels Based on DNA Self-Assembly. Acc. Chem. Res. 2017, DOI: 10.1021/ acs.accounts.6b00524.

(31) Zhan, J. S.; Gao, K.; Chai, R. C.; Jia, X. H.; Luo, D. P.; Ge, G.; Jiang, Y. W.; Fung, Y.-W. W.; Li, L.; Yu, A. C. Astrocytes in Migration. Neurochem. Res. 2017, 42, 272-282.

(32) Hannigan, G.; Leung-Hagesteijn, C.; Fitz-Gibbon, L.; Coppolino, M.; Radeva, G.; Filmus, J.; Bell, J.; Dedhar, S. Regulation of Cell Adhesion and Anchorage-Dependent Growth by a New $\beta 1$ Integrin-Linked Protein Kinase. Nature 1996, 379, 91-96.

(33) Chanput, W.; Mes, J.; Wichers, H. THP-1 Cell Line: An in Vitro Cell Model for Immune Modulation Approach. Int. Immunopharmacol. 2014, 23, 37-45. 